\title{
Simpler than FRESH Filter: A Parametric Approach for Cyclostationary Noise Generation in NB-PLC
}

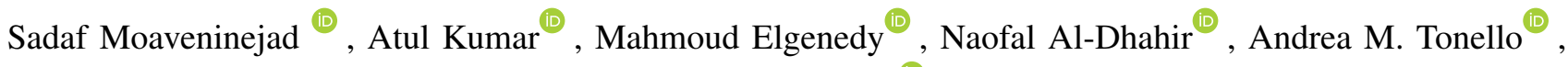 \\ and Maurizio Magarini
}

\begin{abstract}
A key challenge in narrowband power line communications (NB-PLC) is the mitigation of impairments introduced by the correlated cyclostationary noise. The frequencyshift (FRESH) filtering approach has been recently proposed to reproduce a cyclostationary NB-PLC noise with characteristics similar to those obtained from field measurements. In this paper, we use a classification of the noise generated by the FRESH filter into three classes to propose a simple method to produce noise samples with statistics similar to those obtained at its output. The approach consists of parametrized spectral and temporal shapings applied to a white Gaussian noise sequence. We validate our proposed method by comparing its generated noise samples with those obtained using the FRESH filter and using measurements in terms of: $i$ ) normalized mean-squared error between the cyclic auto-correlations; ii) bit error rate.

Index Terms-Impulsive noise, narrowband power line communication (NB-PLC), cyclostationary noise, correlated noise.
\end{abstract}

\section{INTRODUCTION}

A fundamental challenge to achieve reliable transmission in narrowband power line communications (NB-PLC) is to overcome the impairments due to the cyclostationary impulsive noise [1]-[3]. Noise characterization, modelling, and generation are therefore three fundamental steps in the design of NB-PLC systems. Although in many earlier works researchers simply considered additive white Gaussian noise (AWGN), in reality the noise is given by the superposition of different sources [2]. In fact, other noise components exist that are usually characterized by statistical properties with a periodicity that is synchronous with the AC mains cycle [4].

In the context of noise modelling in NB-PLC, an alternative to AWGN is represented by the Middleton's model [5], which has the limitation of providing only a first-order statistical characterization without considering spectral properties. In addition, it does not describe whether the noise is impulsive or smooth [6]. Another model, named as "model \#2" and more specific for NB-PLC, is given by the IEEE standard 1901.2 [7]. It is generated as the sum of three terms: strong impulsive

S. Moaveninejad and M. Magarini are with the Dipartimento di Elettronica, Informazione e Bioingegneria, Politecnico di Milano, Milan, Italy. Email \{sadaf.moaveninejad, and maurizio.magarini\}@polimi.it.

A. Kumar is with the Vodafone Chair Mobile Communications Systems Technische Universitat Dresden, Germany. Email: atul.kumar@tu-dresden.de.

M. Elgenedy and Naofal Al-Dhahir are with Department of Electrical and Computer Engineering, University of Texas at Dallas, Tx USA. Email:\{mahmoud.elgenedy, aldhahir\}@utdallas.edu.

A. Tonello is with Chair of Embedded Communication Systems, University of Klagenfurt 9020 Klagenfurt, Austria. Email: andrea.tonello@aau.at.

Manuscript received XXX, XX, 2019; revised XXX, XX, 2019. short bursts, periodic weaker flat bursts, and background noise. With reference to cyclostationary noise, in [1] a model suitable for single-input single-output NB-PLC systems is proposed, where the random samples are described as zeromean Gaussian random variables with a time-varying variance. A frequency-domain characterization is given in [2], which is important to study the performance of orthogonal frequencydivision multiplexing (OFDM). In [2] a number of $M$ temporal stationary regions is defined, where the noise in each region is obtained as the output of a linear time-invariant (LTI) filter with white Gaussian noise (WGN) at its input. Although this model provides good fitting for the measured NB-PLC noise, it suffers from two drawbacks. First, the number and the boundaries of the temporal regions are based on a visual inspection of the spectogram. Second, the correlation between regions is ignored. In addition, the frequency response of each filter is needed, which increases the system complexity.

An accurate model to generate the correlated cyclostationary NB-PLC noise was proposed in [8], which is based on the use of the frequency-shift (FRESH) filter [9]. In the context of NBPLC, the FRESH filter was originally proposed by Shlezinger et al. [10] to implement linear time-varying filtering at the receiver that exploits the cyclostationary noise generation of [2]. In the FRESH noise approach proposed in [8], a WGN random sequence undergoes $K$ frequency shifts. Each frequency-shifted sequence is applied to the input of an LTI filter with finite impulse response (FIR). The $K$ outputs are then added together to produce the cyclostationary noise. The model was validated in [8] by computing the normalized meansquare-error (NMSE) between the cyclic auto-correlation of the produced sequence and that of the measurements.

The statistics of the noise samples generated by the FRESH filter were studied in [11], where a partition in three classes was proposed. The aim of such a classification was to implement a hybrid system that switches between wireless and PLC links based on the bit error rate (BER) for each of the noise classes. Here, we also use the FRESH filter noise generation with the goal of analyzing the statistics of each class and finding a simple way to generate samples with similar features. Our first contribution is the derivation of the probability density function (PDF) of each class. We show that while one class has a normal PDF, for the other two classes, i.e. strongly and moderate impulsive, a generalized form of the student's $t$-distribution [12] fits better. However, since the PDF knowledge alone is not enough to model the 


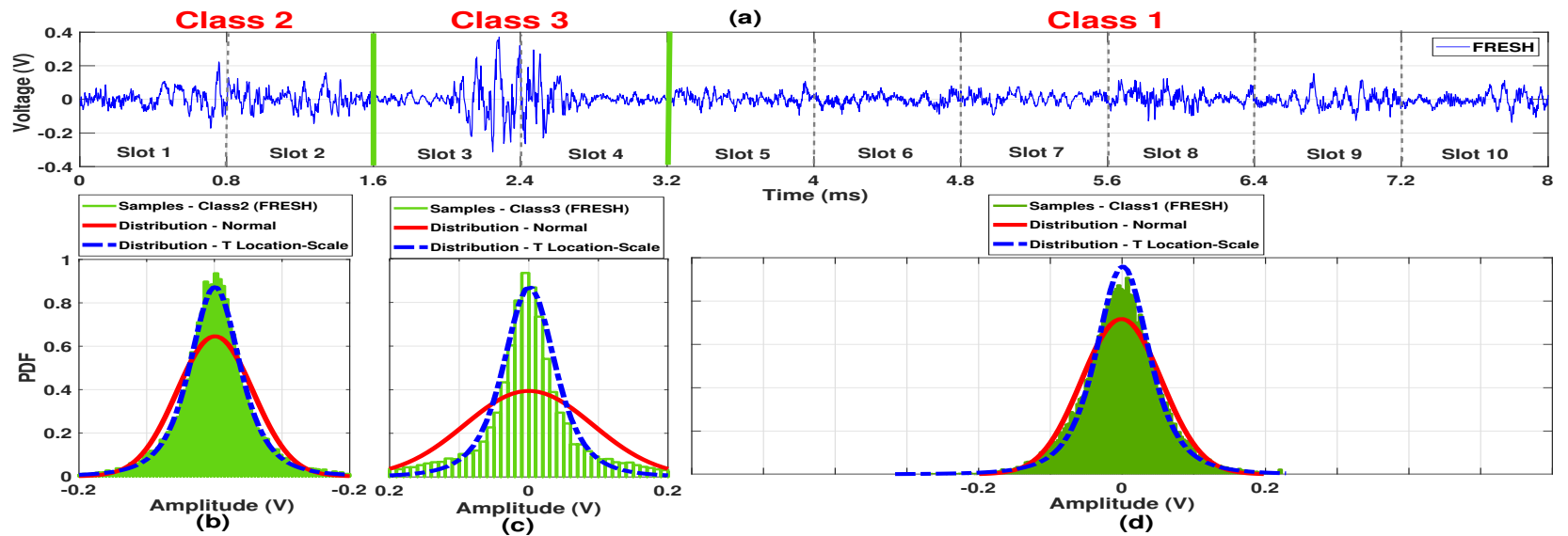

Fig. 1. FRESH noise analysis: (a) classification of FRESH noise in each cycle, (b, c, d) PDF of samples belong to each class and collected from 20 cycles.

impulsive behavior, we also characterize the auto-correlation or, equivalently, the power spectral density (PSD).

As a second contribution of this work, we propose a simplified parametric noise generation approach that can be easily used in computer simulations to produce random samples with statistics similar to those observed at the output of the FRESH filter. The main motivation is that the FRESH filter is based on a high complexity filterbank approach that requires a large number of coefficients for all the FIR filters to achieve a good accuracy. Our approach aims at replacing the bank of FRESH FIR filters with a simple design that requires few parameters. In the proposed scheme each class is generated by shaping the input WGN process with double-sided exponential decaying functions: one in the frequency domain and one in the time domain. Since the frequency-domain shaping is common to all the three classes, it introduces correlation among the NBPLC noise samples in different regions. Note that, this aspect, firstly observed in [8], and here included in the proposed noise generation approach, is not considered in the IEEE 1901.2 standard [7], where the noise sequence is divided into regions each one being modeled independently from the others.

\section{Characterization And Classification OF CYCLOSTATIONARY FRESH NOISE}

Figure 1 (a) shows a snapshot obtained in a cycle of the cyclostationary noise sequence $\left\{z_{n}\right\}$ at the output of the FRESH filter. From a visual inspection, it is possible to identify three distinct regions, each belonging to distinct classes. Similar to [11], and in agreement to what is defined in the IEEE 1901.2 standard [7], in this work we classify the regions into different classes based on the changes of noise statistics in slots with a duration that is equal to that of an OFDM symbol. Based on IEEE 1901.2, each OFDM symbol consists of 320 samples: $N_{\mathrm{fft}}=256$ coming from the computation of the fast Fourier transform (FFT) and $N_{\mathrm{CP}}=64$ from the cyclic prefix.

The classification was done by comparing the difference between the measured noise standard deviation (SD) in each slot $\sigma^{(s)}$ and its minimum $\sigma_{\min }$, taken over all the slots in a cycle, with respect to two empirically chosen thresholds $t h_{1}$ and $t h_{2}$, being $0<t h_{1}<t h_{2}$. The slots where $D^{(s)}=\sigma^{(s)}-\sigma_{\text {min }}$ is in the range from 0 to $t h_{1}$ are considered as class 1 . The indexing of the other two classes was done by assigning an increasing integer as defined by the interval where $D^{(s)}$ falls with respect to the thresholds. Using $\sigma_{\min }$, in place of the average SD over all slots as proposed in [11], improves the classification accuracy. An example of classification results is reported in Fig. 11a), where the last six slots of noise are assigned to class 1 , the first two slots to class 2 , and the remaining slots to class 3 . Note that, other classifications may be undertaken by introducing more parameters and resorting to machine learning approaches [13], [14].

The noise measurements were taken using an oscilloscope with $2.5 \mathrm{MHz}$ sampling rate. To develop the FRESH model a downsampled version of the noise sequence sampled at $400 \mathrm{kHz}$ was considered, which corresponds to the sampling rate considered in the CENELEC-A and CENELEC-B frequency bands. Here, the FRESH filter was used to generate $N_{\text {cycle }}=20$ noise cycles of duration $T=\frac{1}{2 \times 62.5}=8 \mathrm{~ms}$, which is half of the inverse of $62.5 \mathrm{~Hz}$, i.e. the AC cycle frequency. With sampling frequency equal to $400 \mathrm{kHz}$, there are 3200 samples in each cycle. Since the length of the slot is the same as that of one OFDM symbol, each slot contains $N_{\text {sample }}=320$ samples. Therefore, in each cycle there are $N_{\text {slot }}=10$ slots and each class $i$, with $i=1,2,3$, will contain $\left(n_{s}\right)_{i}$ slots so that $\sum_{i=1}^{3}\left(n_{s}\right)_{i}=10$. Considering $N_{\text {cycle }}$ cycles, the total number of samples in each class $i$ is equal to $\left(n_{s}\right)_{i} \times N_{\text {sample }} \times N_{\text {cycle }}$. After assigning each noise slot to a specific class, we collected all noise samples from $N_{\text {cycle }}=20$ cycles belonging to that class. Classes 2 and 3 have 12800 samples each while class 1 contained 38400 samples. The estimated PDF and the analyzed spectral and temporal behavior of each class were then used to develop a model for generating noise samples similar to that at the output of FRESH filter as described next.

\section{FRESH NOISE PDF ESTIMATION}

Figures 1 (b), (c), and (d) report the estimated PDFs for the noise samples generated by the FRESH filter and classified according to the three classes described in Sec. III In each figure, the red curve is associated with the normal distribution that has been obtained by using the mean and variance estimated from the samples belonging to the respective class.

Figure 11 d) demonstrates that the normal distribution fits the PDF of class 1 , hence this class could be considered as 


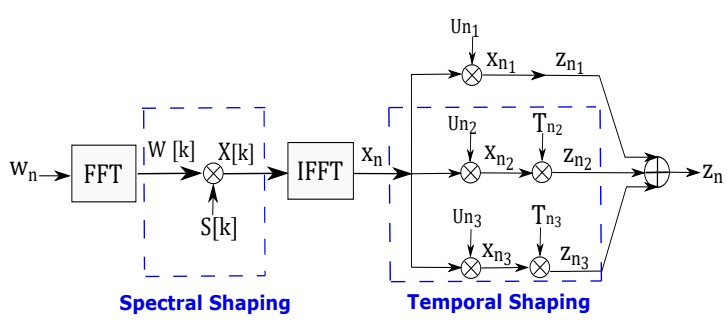

Fig. 2. Block diagram of noise model generation.

Gaussian noise process. As can be observed from Fig. 1 (b), the behavior of class 2 is between Gaussian and impulsive. Here, we consider class 2 as moderate impulsive. However, we observed that in different cycles the behavior of class 2 may become alternatively closer to be Gaussian or to be impulsive. Similarly, the big difference between the variance of class 3 and normal distribution in Fig. 1(c) implies that this class could be considered as strongly impulsive noise. With these observations in mind, we need to find the distribution that matches the PDF and the variance of classes 2 and 3. We used the distribution fitting app of MATLAB ${ }^{\circledR}$ to interactively fit different distributions to the samples of each class and find the best fitting. The general form of the $t$-distribution with three parameters location-scale family, known as $t$ locationscale distribution fits well the PDF of classes 2 and 3. This is evident from the blue curves in Figs. 11(b) and (c). The $t$-distribution is symmetric and bell-shaped, like the normal distribution, but used in situations with known mean $\mu$ and unknown standard deviation. Its PDF is given by

$$
p(x \mid \nu, \mu, \sigma)=\frac{\Gamma\left(\frac{\nu+1}{2}\right)}{\sigma \sqrt{\nu \pi} \Gamma\left(\frac{\nu}{2}\right)}\left[\frac{\nu+\left(\frac{x-\mu}{\sigma}\right)^{2}}{\nu}\right]^{-\left(\frac{\nu+1}{2}\right)},
$$

where $\Gamma(\cdot)$ is the Gamma function and $\nu, \mu$, and $\sigma$ are known as the shape, location, and scale parameters, respectively [15].

It is worth observing that the samples of the measured noise are not independent and a correlation exists among them. Samples generated by the FRESH filter are also correlated and this is not considered in the PDF. Therefore, the PDF in (1) can be used in the analysis of the system to develop a lower bound on performance where the noise samples are independent. To address this issue, we developed another solution to generate correlated noise samples based on spectra-temporal shaping (ST-shaping), which is simpler than FRESH filtering and is easier to be adapted to the distribution of real measurements.

\section{Parametric Cyclostationary Noise Generation}

In our proposed parametric approach, we used the temporal and the spectral characterization of each noise class by considering all the samples generated by the FRESH filter in the 20 cycles. The block diagram of the proposed parametric approach is shown in Fig. 2. A sequence $\left\{w_{n}\right\}$ of zero mean complex ${ }^{1}$ WGN samples with unitary variance and length $N_{\text {sample }} \times N_{\text {slot }}$ is generated, i.e. of the same duration

\footnotetext{
${ }^{1}$ We used a complex WGN as a general case of a complex baseband transmission. However, according to the multi-tone real baseband transmission defined in the IEEE 1901.2 standard, transmitted signal is always real where tones are enabled only on the selected band. In this case, only real part of the noise is used.
}

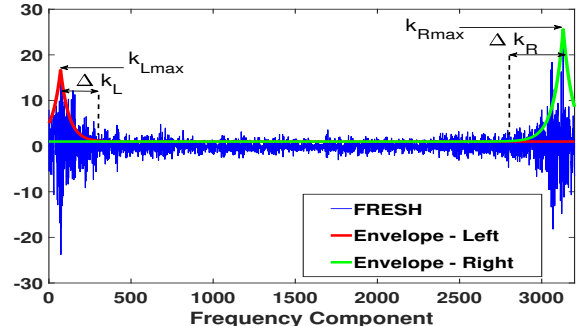

Fig. 3. Example of spectral shaping in one cycle based on the envelope of FFT of FRESH noise.

as the output of the FRESH filter in one cycle. After that, the noise samples are shaped in the frequency domain and in the temporal domain as illustrated in Fig. 2. Spectral shaping over all the samples in each cycle is implemented in the first part of the scheme. Since the FFT computation, followed by frequency-domain weighting, and the inverse FFT (IFFT) are linear operations, the resulting random sequence $\left\{x_{n}\right\}$ is still Gaussian. By this, we inherently assume that the PSD is almost the same for the three classes. Hence, at this point all the samples have the class 1 statistics. The main difference between different classes is in the time domain. Consequently, we need to distribute samples in different slots to the appropriate class. Since class 2 and 3 are non-Gaussian, further temporal shaping is required to generate noise samples with the same statistics of those at the FRESH filter output.

Spectral and temporal shaping are realized by means of parametric functions that approximate the envelopes of the observed noise realizations in the respective domains. For both of them, we propose a double-sided decaying exponential function with a parametrization that is discussed in the next two subsections. As observed in [1], due to the impulsiveness of the noise, several local minima and maxima are observed in a single realization. Therefore, in each cycle, two points are selected as the most significant ones from each shape to estimate the parameters for the corresponding envelope.

\section{A. Spectral Shaping}

Figure 3 reports an example of the magnitude of the FFT computed in a cycle of the complex noise produced at the output of the FRESH filter. It can be observed that two nonsymmetric double-sided decaying exponential functions can be used to approximate the envelope. The decay constant for each function can be obtained by interpolating two points chosen from the computed FFT magnitude. The indexes of such two points are chosen as follows: the first, i.e. $k_{L_{\max }}$, is the frequency component that has the highest amplitude in the leftside curve $Y_{k_{L_{\max }}}$; the second is $k_{L_{\min }}=k_{L_{\max }}+\Delta k_{L}$. Similarly, $k_{R_{\max }}$ is the frequency component with the highest amplitude in the right-side curve and $k_{R_{\min }}=k_{R_{\max }}-\Delta k_{R}$ is the other point selected for the envelope of the right-side curve with amplitudes $Y_{k_{R_{\max }}}$, and $Y_{k_{R_{\min }}}$, respectively. Here, we choose $\Delta k_{L}=\Delta k_{R}=125$ samples. The length of $\Delta k_{L, R}$ affects the width of the double-sided decaying exponentials in $X[k]$ and, consequently, the slope of the PSD. The spectral envelopes of noise samples generated by FRESH filtering in one cycle are modeled in parametric way as: 


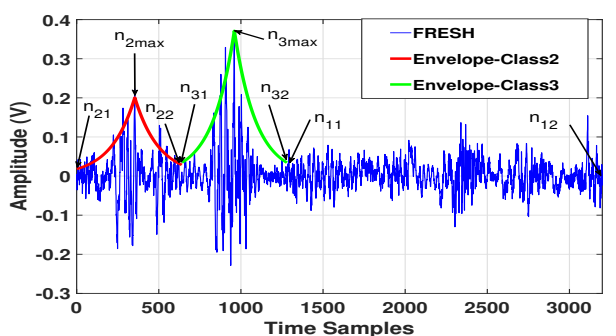

Fig. 4. Double-sided envelopes of the temporal shaping of class 2 and class 3 for the noise sequence generated by the FRESH filter in one cycle.

$$
\begin{aligned}
& X[k]=S[k] W[k], S[k]=S[k]_{L}+S[k]_{R}+C \\
& S[k]_{L}=A_{L} e^{B_{L}\left|k-k_{L_{\max }}\right|}, S[k]_{R}=A_{R} e^{-B_{R}\left|k-k_{R_{\max }}\right|} \\
& B_{L}=\frac{\ln \left(Y_{k_{L_{\min }}}\right)-\ln \left(Y_{k_{L_{\max }}}\right)}{k_{L_{\min }}-k_{L_{\max }}}, B_{R}=\frac{\ln \left(Y_{k_{R_{\max }}}\right)-\ln \left(Y_{k_{R_{\min }}}\right)}{k_{R_{\max }}-k_{R_{\min }}},
\end{aligned}
$$

where $k=1, \ldots, 3200, \ln (\cdot)$ is the natural logarithm, and

- $A_{L}$ and $A_{R}$ are the $y$-intercepts of the left-side and rightside graphs, respectively, i.e. $A_{L}=Y_{k_{L_{\max }}}$ and $A_{R}=Y_{k_{R_{\max }}}$;

- $B_{L}$ and $B_{R}$ are the slopes of the exponential envelopes $S[k]_{L}$ and $S[k]_{R}$, respectively. Since samples are complex, there are two non-symmetric exponential functions, while in case of real samples the two are symmetric;

- $C$ is the constant tail of the envelope, here set equal to 1 .

\section{B. Temporal Shaping}

In this subsection, the temporal envelopes of classes 2 and 3 are evaluated in a way similar to that used for the rightside envelope of the spectral shaping $S[k]_{R}$. For each class $i=2,3$, the $y$-intercept of the samples in the time domain and the slope of the exponential are indicated by $A_{i}$ and $B_{i}$, respectively. Three points are chosen from each class to define the envelope for the temporal shaping. These are the starting point $\left(n_{i 1}, y_{n_{i 1}}\right)$, the maximum amplitude point $\left(n_{i_{\max }}, y_{n_{i_{\max }}}\right)$, and the ending point $\left(n_{i 2}, y_{n_{i 2}}\right)$. Regarding class 1 , there is no need to do temporal shaping since it is already Gaussian. For the temporal shaping, with reference to Fig. 2, the following parameters are defined:

$$
\begin{aligned}
& U_{n_{i}}=\left(u_{n-n_{i 1}}-u_{n-n_{i 2}}\right), \quad x_{n_{i}}=x_{n} U_{n_{i}}, \quad i=1,2,3 \\
& z_{n_{1}}=x_{n_{1}}, \quad z_{n_{i}}=x_{n_{i}} T_{n_{i}}, \quad i=2,3 \\
& T_{n_{i}}=A_{i} e^{-B_{i}\left|n-n_{i 2}\right|}, B_{i}=\frac{\ln \left(y_{n_{i_{\max }}}\right)-\ln \left(y_{n_{i 1}}\right)}{n_{i_{\max }}-n_{i 1}}, A_{i}=y_{n_{i 2}},
\end{aligned}
$$

where $u_{n}$ is the unit step function. Each portion of the output is assigned to one of the the three classes by defining its starting point $n_{i 1}$ and its ending point $n_{i 2}$, which could be modified to change the position, duration, and even the presence of that class in a cycle. Note that in order to use this model, a normalization of the maximum amplitude of the random sequence to 1 is required before multiplying by the doublesided decaying function to obtain the desired peak amplitude level. Figure 4 shows the time-domain envelopes of classes 2 and 3 for the noise samples generated by the FRESH filter. It is worth mentioning again that in different cycles, the behavior of class 2 may be closer to Gaussian or impulsive. Moreover, the strong impulses of class 3 may become worse or smoother. Keeping this fact in mind, the impulsive or Gaussian behavior of each class $i$ is adjustable through $A_{i}$ and $B_{i}$.

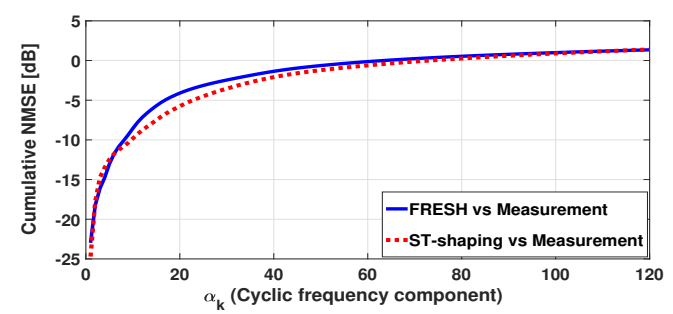

Fig. 5. Cumulative NMSE over cyclic frequency components

\section{Simulation AND Analysis}

A cyclostationary signal $z_{n}$ of period $N$ has auto-correlation $R_{z_{n, l}=}=\mathbb{E}\left\{z_{n+l} z_{n}^{*}\right\}$ that is periodic of the same period, i.e. $R_{z_{n, l}}=R_{z_{n+N, l}}$ [16]. Let $\hat{z}_{n}$ be the measured cyclostationary noise with period $N=N_{\text {sample }} \times N_{\text {slot }}$. The periodic autocorrelation can be represented by its Fourier series (FS) as $\sum_{k=0}^{N-1} R_{\hat{z}_{l}}^{\alpha_{k}} e^{j 2 \pi \alpha_{k} n}[16$. The $k$ th coefficients of the FS is $R_{\hat{z}_{l}}^{\alpha_{k}}=\frac{1}{N} \sum_{n=0}^{N-1} R_{\hat{z}_{n, l}} e^{-j 2 \pi \alpha_{k} n}$, which is referred to as cyclic auto-correlation. Moreover, $R_{\tilde{z}_{l}}^{\alpha_{k}}$ represents the strength of the harmonic component with the cyclic frequency $\alpha_{k}=k / N$, that contributes to the periodic correlation function. Figure 5 depicts the cumulative NMSE between the cyclic auto-correlation of the samples generated by ST-shaping $\left(R_{z_{l}}^{\alpha_{k}}\right)_{\text {ST-shaping and original measurements that, for the } k \text { th }}$ cyclic frequency component is defined as [8]:

$$
\left(\mathrm{NMSE}_{\alpha_{k}}\right)_{\mathrm{ST} \text {-shaping }}=\frac{\sum_{l}\left|R_{\hat{z}_{l}}^{\alpha_{k}}-\left(R_{z_{l}}^{\alpha_{k}}\right)_{\mathrm{ST} \text {-shaping }}\right|^{2}}{\sum_{l}\left|R_{\hat{z}_{l}}^{\alpha_{k}}\right|^{2}} .
$$

The cumulative NMSE is obtained by summing over all the $\alpha_{k}$ 's, $k=0,1, \ldots, N-1$ and normalizing by the total energy of cyclic auto-correlation related to the measured noise samples. The figure reports also the cumulative NMSE between the cyclic auto-correlation of the samples generated with FRESH filter $\left(R_{z_{l}}^{\alpha_{k}}\right)_{\text {FRESH }}$ and original noise. Here, the FRESH filter generates noise by using 19 filter branches and each filter consists of 50 coefficients. It was shown in [8] that the initial value for cumulative NMSE at $\alpha_{0}$ depends on the number of coefficients in a FIR filter while the slope of the linear segment of the curve depends on the number of branches. Figure 6 shows that the BER of the proposed ST-shaping is comparable with FRESH filtering but closer to that of real measurements. The simulation is done for an OFDM NB-PLC system with binary phase-shift keying (BPSK) modulation and with a multipath channel measured over low voltage lines in the laboratory. Figure 7 shows a realization of noise in one cycle. Similar behavior is shown in the following cycles.

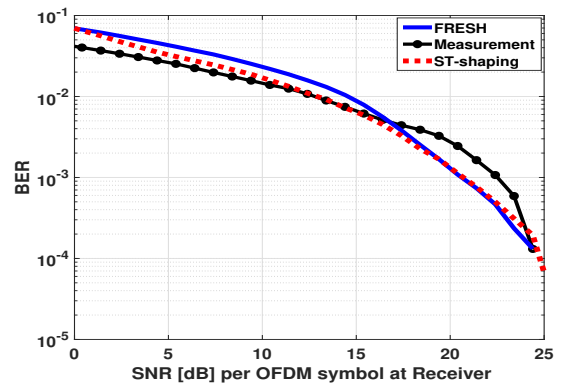

Fig. 6. BER comparison in presence of noise samples obtained by: measurement, FRESH filtering, ST-shaping. 
TABLE I

PARAMETERS FOR SPECTRAL SHAPING IN ONE CYCLE OF 3200 SAMPLES.

\begin{tabular}{|c|c|c|c|c|l|}
\hline $\begin{array}{c}\text { Envelope } \\
\text { side }\end{array}$ & $A=Y_{k_{\max }}$ & $B$ & $k_{\max }$ & $k_{\min }$ & $Y_{k_{\min }}$ \\
\hline Left & 15.6 & -0.019 & 71 & 196 & 1.38 \\
\hline Right & 24.5 & 0.017 & 3131 & 3006 & 2.9 \\
\hline
\end{tabular}

TABLE II

PARAMETERS FOR TEMPORAL SHAPING OF SAMPLES IN EACH CLASS.

\begin{tabular}{|c|c|c|c|c|c|c|c|}
\hline $\begin{array}{c}\text { Class } \\
\text { index } \\
\mathbf{i}\end{array}$ & $A_{i}=y_{n_{i_{\max }}}$ & $B_{i}$ & $n_{i 1}$ & $n_{i_{\max }}$ & $n_{i 2}$ & $y_{n_{i 1}}$ & $y_{n_{i 2}}$ \\
\hline $\mathbf{2}$ & 0.2005 & 0.0068 & 1 & 354 & 640 & 0.0181 & 0.0105 \\
\hline $\mathbf{3}$ & 0.3713 & 0.0074 & 641 & 960 & 1280 & 0.0345 & 0.0198 \\
\hline
\end{tabular}

TABLE III

PARAMETERS OF THE DISTRIBUTIONS IN EACH CLASS.

\begin{tabular}{|c|c|c|c|c|c|}
\cline { 2 - 6 } \multicolumn{1}{c|}{} & \multicolumn{2}{c|}{ Normal } & \multicolumn{2}{c|}{ T Location-Scale } \\
\hline \multirow{2}{*}{ Class } & Mean & Variance & Location & Scale & Shape \\
& $\mu$ & $\sigma$ & $\mu$ & $\sigma$ & $\nu$ \\
\hline $\mathbf{1}$ & $8.66 \times 10^{-5}$ & 0.0017 & $-7.73 \times 10^{-5}$ & 0.034 & 6 \\
\hline $\mathbf{2}$ & $11 \times 10^{-5}$ & 0.0022 & $161.9 \times 10^{-5}$ & 0.031 & 3 \\
\hline $\mathbf{3}$ & $29 \times 10^{-5}$ & 0.0055 & $100.3 \times 10^{-5}$ & 0.03 & 1.5 \\
\hline
\end{tabular}

Examples for the parameters of the ST-shaping approach in one cycle are given in Tables I and II. The same process for obtaining parameters $A_{L}, A_{R}, A_{2}$, and $A_{3}$ was repeated in each of the 20 cycles. From the analysis of long sequences of noise samples at the output of the FRESH filter we observed that each parameter can be described by a normal distribution. Consequently, it is possible to generate noise samples for several realization cycles by drawing random numbers from such normal distributions. Specifically, the normal distributions for the amplitudes of the spectral shaping are described by $A_{L} \sim N(18,8)$ and $A_{R} \sim N(17,11)$, being the first and the second parameter the mean and the variance, respectively. Similarly, for the amplitudes of the temporal shaping we have $A_{2} \sim N(0.2141,0.0031)$ and $A_{3} \sim N(0.243,0.005)$. Note that these amplitudes and other parameters of the ST-shaping could vary in a way such that the slope of the corresponding envelope remains fixed to the value given in Table I or Table II Using this ST-shaping approach, we generate correlated impulsive noise for NB-PLC in a period equal to $8 \mathrm{~ms}$. The starting and ending points of class 1 are set to $n_{11}=(4 \times 320)+1=1281$ and $n_{12}=10 \times 320=3200$. However, since class 1 can be considered as background noise, its starting and ending points are defined by the first and last sample in each cycle. Also, for the other two classes, the parameters given in Table [I] can be modified to change the position, duration, and magnitude of each class. Table III] reports values for different parameters of the normal and $t$-student distributions that fit the noise samples for each class of the FRESH filter.

\section{CONCLUSION}

We proposed a parametric method to generate cyclostationary noise in NB-PLC. Our method is simpler than the FRESH filtering method, which was defined to reproduce a cyclostationary noise with the same statistical properties as that obtained from field measurements. FRESH filtering achieves high accuracy but it is characterized by complexity requiring large number of parameters. Therefore, a simpler model as the one proposed here can be more suitable to evaluate NBPLC performance by computer simulations. Our proposed method is based on the analysis of the cyclostationary noise

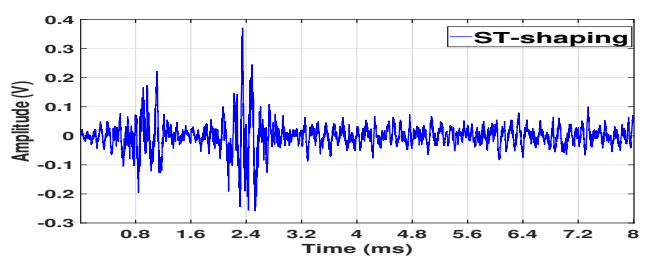

Fig. 7. Waveform generated by ST-shaping in one cycle.

generated by the FRESH filter and its division into nonoverlapping temporal regions referred to as noise classes. Such a classification is used together with the spectral and temporal analysis of the random sequence generated by the FRESH filter to tune the parameters required to shape a white Gaussian sequence and obtain either Gaussian or impulsive correlated samples. Numerical results are used to compare the NMSE and BER that were obtained with the noise samples from the proposed ST-shaping, those generated by the FRESH filter, and by the original measurements. The main advantage of our new simplified approach is its high flexibility, where by changing few parameters it is possible to adjust the desired degree of impulsiveness and Gaussianity.

\section{REFERENCES}

[1] M. Katayama, T. Yamazato, and H. Okada, "A mathematical model of noise in narrowband power line communication systems," IEEE J. Sel. Areas Commun., vol. 24, pp. 1267-1276, July 2006.

[2] M. Nassar, A. Dabak, I. H. Kim, T. Pande, and B. L. Evans, "Cyclostationary noise modeling in narrowband powerline communication for smart grid applications," in Proc. of ICASSP, pp. 3089-3092, March 2012.

[3] M. Antoniali, F. Versolatto, and A. M. Tonello, "An experimental characterization of the PLC noise at the source," IEEE Trans. Power Del., vol. 31, pp. 1068-1075, June 2016.

[4] M. Elgenedy, M. Sayed, M. Mokhtar, M. Abdallah, and N. Al-Dhahir, "Interference mitigation techniques for narrowband powerline smart grid communications," in 2015 IEEE International Conference on Smart Grid Communications (SmartGridComm), pp. 368-373, Nov 2015.

[5] D. Middleton, "Statistical-physical models of electromagnetic interference," IEEE Trans. Electromagn. Compat., no. 3, pp. 106-127, 1977.

[6] L. Lampe, A. M. Tonello, and T. G. Swart, Power Line Communications: Principles, Standards and Applications from multimedia to smart grid. John Wiley \& Sons, 2016.

[7] "IEEE Standard for Low-Frequency (less than $500 \mathrm{kHz}$ ) Narrowband Power Line Communications for Smart Grid Applications,' IEEE Std 1901.2-2013, pp. 1-269, Dec 2013.

[8] M. Elgenedy, M. Sayed, A. El Shafie, I. H. Kim, and N. Al-Dhahir, "Cyclostationary noise modeling based on frequency-shift filtering in NBPLC," in Proc. of GLOBECOM, pp. 1-6, Dec 2016.

[9] W. A. Gardner, "Cyclic wiener filtering: theory and method," IEEE Trans. Commun., vol. 41, pp. 151-163, Jan 1993.

[10] N. Shlezinger and R. Dabora, "Frequency-shift filtering for OFDM signal recovery in narrowband power line communications," IEEE Trans. Commun., vol. 62, pp. 1283-1295, April 2014.

[11] S. Moaveninejad, A. Kumar, M. Elgenedy, M. Magarini, N. Al-Dhahir, and A. Tonello, "Gaussian-Middleton classification of cyclostationary correlated noise in hybrid MIMO-OFDM WiNPLC," in Proc. ICC, pp. 17, 2019.

[12] K. L. Lange, R. J. Little, and J. M. Taylor, "Robust statistical modeling using the t distribution," Journal of the American Statistical Association, vol. 84, no. 408, pp. 881-896, 1989.

[13] A. M. Tonello, N. A. Letizia, D. Righini, and F. Marcuzzi, "Machine learning tips and tricks for power line communications," IEEE Access, vol. 7, pp. 82434-82452, 2019.

[14] D. Righini and A. M. Tonello, "Automatic clustering of noise in multiconductor narrow band PLC channels," in Proc. of ISPLC, pp. 1-6, 2019.

[15] C. M. Bishop, Pattern recognition and machine learning. Springer, 2006.

[16] F. J. Cañete, L. Díez, J. A. Cortés, and J. T. Entrambasaguas, "Cyclic signals and systems in power line communications," IEEE Access, vol. 7, pp. 96799-96817, 2019. 